\title{
Glucosamine and Chondroitin Sulfate: Is There Any Scientific Evidence for Their Effectiveness as Disease-Modifying Drugs in Knee Osteoarthritis Preclinical Studies?-A Systematic Review from 2000 to 2021
}

\author{
Silvia Fernández-Martín ${ }^{1, *} \mathbb{C}$, Antonio González-Cantalapiedra ${ }^{1,2} \mathbb{C}$, Fernando Muñoz ${ }^{1,2} \mathbb{D}$, \\ Mario García-González ${ }^{1}$ (D) María Permuy $^{2}$ (D) and Mónica López-Peña ${ }^{1,2}$ (D) \\ 1 Anatomy, Animal Production and Veterinary Clinical Sciences Department, Veterinary Faculty, \\ Campus Universitario s/n, Universidade de Santiago de Compostela, 27002 Lugo, Spain; \\ antonio.cantalapiedra@usc.es (A.G.-C.); fernandom.munoz@usc.es (F.M.); mariog.gonzalez@usc.es (M.G.-G.); \\ monica.lopez@usc.es (M.L.-P.) \\ 2 Ibonelab S.L., Laboratory of Biomaterials, Avda. da Coruña, 500 (CEI-NODUS), 27003 Lugo, Spain; \\ permuy@ibonelab.com \\ * Correspondence: silvia.fernandez.martin@usc.es; Tel.: +34-982-82-2631
}

Citation: Fernández-Martín, S.; González-Cantalapiedra, A.; Muñoz, F.; García-González, M.; Permuy, M.; López-Peña, M. Glucosamine and Chondroitin Sulfate: Is There Any Scientific Evidence for Their Effectiveness as Disease-Modifying Drugs in Knee Osteoarthritis Preclinical Studies?-A Systematic Review from 2000 to 2021. Animals 2021, 11, 1608. https://doi.org/ $10.3390 /$ ani11061608

Academic Editors: Rosalia Crupi and Enrico Gugliandolo

Received: 17 April 2021

Accepted: 27 May 2021

Published: 29 May 2021

Publisher's Note: MDPI stays neutral with regard to jurisdictional claims in published maps and institutional affiliations.

Copyright: (c) 2021 by the authors. Licensee MDPI, Basel, Switzerland. This article is an open access article distributed under the terms and conditions of the Creative Commons Attribution (CC BY) license (https:/ / creativecommons.org/licenses/by/ $4.0 /)$.
Simple Summary: Osteoarthritis is the most common progressive joint disease diagnosed in companion animals and its management continues to be a significant challenge. Nutraceuticals have been widely investigated over the years in the treatment of osteoarthritis and among them, glucosamine and chondroitin sulfate treatments are probably the most common therapies used in veterinary management. However, heterogeneous results were obtained among animal studies and the evidence of their efficacy is still controversial. Animal models have a crucial role in studying the histological changes and evaluating the therapy efficacy of different drugs. Consequently, we consider it may be of interest to evaluate the effectiveness of the most representative nutraceuticals in experimental animal studies of osteoarthritis. In this systematic review, we found a large inconsistency among the experimental protocols, but a positive cartilage response and biochemical modulation were observed in half of the evaluated articles, mainly associated with pre-emptive administrations and with some therapies' combinations. Even though some of these results were promising, additional data are needed to draw solid conclusions, and further studies evaluating their efficacy in the long term and focusing on other synovial components may be needed to clarify their function.

Abstract: Glucosamine and chondroitin sulfate have been proposed due to their physiological and functional benefits in the management of osteoarthritis in companion animals. However, the scientific evidence for their use is still controversial. The purpose of this review was to critically elucidate the efficacy of these nutraceutical therapies in delaying the progression of osteoarthritis, evaluating their impact on the synovial knee joint tissues and biochemical markers in preclinical studies by systematically reviewing the last two decades of peer-reviewed publications on experimental osteoarthritis. Three databases (PubMed, Scopus and, Web of Science) were screened for eligible studies. Twentytwo articles were included in the review. Preclinical studies showed a great heterogeneity among the experimental designs and their outcomes. Generally, the evaluated nutraceuticals, alone or in combination, did not seem to prevent the subchondral bone changes, the synovial inflammation or the osteophyte formation. However, further experimental studies may be needed to evaluate their effect at those levels. Regarding the cartilage status and biomarkers, positive responses were identified in approximately half of the evaluated articles. Furthermore, beneficial effects were associated with the pre-emptive administrations, higher doses and, multimodality approaches with some combined therapies. However, additional studies in the long term and with good quality and systematic design are required. 
Keywords: animal models; biochemical markers; cartilage; chondroitin sulfate; glucosamine; nutraceuticals; osteoarthritis

\section{Introduction}

Osteoarthritis (OA) is a heterogeneous chronic disease that involves all tissues in the synovial joints. It is usually characterized by progressive cartilage damage, subchondral bone changes, osteophyte formation, synovial inflammation and the secretion of inflammatory mediators [1,2]. At present, it is most common progressive joint disease diagnosed in companion animals and its management continues to be a significant challenge [3]. Lameness, stiffness and chronic pain resulting from the OA pathology have a negative impact on the quality of life of the affected animals [4]. Additionally, OA pain is frequently mishandled in animals, and consequently, some clinical cases may result in premature euthanasia [5].

At present, there is hardly any accurate epidemiological data available of this disease in the different animal species [6]. In sport horses, OA is one of the most prevalent and disabling diseases in sport horses, fundamentally affecting the metacarpophalangeal joint and causing chronic and painful lameness as well as an important economic loss in the equine industry [7]. Furthermore, a recent study has reported a noteworthy prevalence of cervical OA in jumping horses. More specifically, a moderate to severe OA was observed at C6-7 in $25 \%$ of the studied population [8]. In dogs, OA is highly prevalent with reports of around $20 \%$ of the canine population over a year-old [9]. Nevertheless, subsequent studies reported lower values, as observed by Anderson et al. [3] and $\mathrm{O}^{\prime}$ Neill et al. [10], who estimated an OA prevalence of $2.5 \%$ or $6.6 \%$ in primary-care practices in the UK. Generally, large-breed dogs developed initial and more severe clinical signs of OA [4]. However, early symptoms may be overlooked by the owner or considered normal, thus the joint disease is usually diagnosed at a later stage [3]. In cats, it is a very common joint disease, especially in older cats. In relation to this, a previous study reported an OA prevalence of around $61 \%$ at over 6 years of age [11]. OA in cats seems to be related to behavioural changes such as decreased mobility and less grooming [11]. However, it is important to highlight the underdiagnosis of the disease associated with the lack of signs such as lameness and a lower radiographic identification. This is in addition to its difficult physical examination by clinicians [12]. Furthermore, the treatment of OA is a major challenge in this specie, related to reduced availability of drugs as well as increased adverse effects and complications [13].

For many years, the available therapeutic options for OA management were focused on inflammation relief and pain control and were basically restricted to the use of nonsteroidal anti-inflammatory drugs and analgesics. However, their chronic administration was limited by their deleterious systemic side effects [6]. Currently, there is no ideal drug capable to reverse or stop the progression of the OA and for that purpose, numerous therapeutic agents have been widely researched for their potential role in targeting the underlying pathology of OA with various levels of efficacy [14]. Nutraceuticals, also classically called chondroprotectors, have been widely analysed over the years in the treatment of OA in companion animals. Among them, glucosamine and chondroitin sulfate treatments are probably the most commonly used in the veterinary management of OA [4]. These dietary supplements have been proposed to promote the cartilage and periarticular bone health status [15] and their effectiveness in the OA progression has been thoroughly tested in experimental research. However, heterogeneous results were obtained in different animal studies and their function as disease-modifying drugs is still controversial. Some published clinical trials in dogs treated with glucosamine and chondroitin sulfate, reported positive clinical effects with significant pain relief [16], whereas in other publications, no significant differences were found between treated and untreated dogs $[17,18]$. 
At present, there are multiple reviews focusing on the effects of dietary supplements in clinical signs of companion animal OA, as an attempt to clarify their effectiveness in the OA management $[4,19-21]$. In this context, a previous literature review studied some nutraceutical effects in different OA animal models [22]. However, in that case, they fundamentally focused on determining the most suitable animal model to examine the potential beneficial roles of different nutraceuticals such as vitamins, avocado and soybean, polyphenols and glycosaminoglycans, among others. To the best of our knowledge, no current systematic reviews have evaluated their impact on the structural OA changes in animal models. Preclinical animal models offer a great opportunity to better understand the pathophysiology of the OA and to evaluate the therapeutic response [23]. Consequently, we consider it may be of interest to review, synthesise and evaluate the effectiveness of the most representative nutraceuticals in different animal model studies, given the available scientific data.

The purpose of this review was to critically elucidate the efficacy of glucosamine and chondroitin sulfate therapies in delaying the progression of $\mathrm{OA}$, evaluating their impact on the synovial knee joint tissues and biochemical markers in preclinical studies by systematically reviewing the last two decades of peer-reviewed publications on experimental OA.

\section{Materials and Methods}

\subsection{Protocol and Search Strategy}

This systematic review followed the Preferred Reporting Items for Systematic Reviews and Meta-Analyses (PRISMA) guidelines [24]. The literature search was performed using the following online scientific databases: PubMed, Web of Science and Scopus. The studies were identified using combinations of the following terms: "osteoarthritis", "animal models", "glucosamine" and/or "chondroitin sulfate" as keywords.

The inclusion criteria were as follows:

- Experimental preclinical studies in animal models of OA which focused on the structural effect of glucosamine and chondroitin sulfate on the knee synovial joint tissues and biochemical markers.

- Studies that included outcome measures by using gross, histologic, histomorphometric, biochemical and/or imaging techniques.

- Articles written in English language.

- Studies published in international peer-reviewed journals between 2000 and February 2021.

The exclusion criteria were articles written in other languages, reviews, abstracts, book chapters, in vitro studies and clinical human or animal trials. Furthermore, articles that did not include an OA animal model, with intraarticular administrations of therapies, or reports in which the joint of interest was not the knee, were also excluded.

\subsection{Study Selection and Data Extraction}

The titles, abstracts and the full text of the articles, identified by Internet searches, were screened by two reviewers (S.F.-M. and A.G.-C.). The eligible papers were carefully read, and the following data were extracted: author and year of publication, animal model (breed, species, gender, age and number of animals), OA model according with its cause, nutraceuticals therapy including type, dosage, frequency and the administration route, baseline, time of sacrifice, outcome measures and main results.

Subsequently, we independently evaluated the main outcomes determined in the included articles based on the type of nutraceuticals studied and their structural effects on the cartilage, subchondral bone and synovial membrane. Additionally, their influence on the osteophyte formation and biomarkers fluctuations was taken into account. We classified the outcomes as positive effect (+), negative effect or no effect $(-)$ and unclear effect or no significant effect (?). In addition, we marked as not included (x) when the parameters were not evaluated in the study. Regarding the initial administration of the therapies, we classified them as pre-emptive therapies if they were administered before OA induction, 
early therapies when they were administered between OA induction and 14 days post-OA induction, and delayed therapies if the baseline was more than 14 days post-OA induction.

In addition, the studies were classified based on the therapy duration, into shortterm therapies ( $\leq 8$ weeks), intermediate-term therapies ( $>8$ to $<24$ weeks) and long-term therapies ( $\geq 24$ weeks). In the studies where different therapy durations were included, we selected the longest term.

\subsection{Quality Assessments and Risk of Bias}

The quality and risk-of-bias assessments were performed by two independent authors (S.F.-M. and A.G.-C.), and any discrepancies were resolved by team consensus with all the authors. To assess the quality of the animal studies, we analysed all the included manuscripts using the updated guidelines for reporting animal research: the ARRIVE guidelines (Animal Research: Reporting of In Vivo Experiments) [25]. More specifically, we used the "Compliance Questionnaire" in order to evaluate whether the manuscripts complied with the ARRIVE Essential 10: Study design, sample size, inclusion and exclusion criteria, randomisation, blinding, outcome measures, statistical methods, experimental animals, experimental procedures and results. We checked each of the 10 items and assigned the category of "reported" if the item was completely reported, "not reported" if it was not reported and "unclear" if it partially reported or if insufficient details were provided.

The risk of bias was assessed using the Systematic Review Centre for Laboratory animal Experimentation (SYRCLE) tool for animal studies in order to assign a judgement of low, high or unclear risk of bias to each of the 10 items included in the checklist: sequence generation, baseline characteristics, allocation concealment, random housing, blinding caregivers and/or investigator, random outcome assessment, blinding outcome assessor, incomplete outcome data, selective outcome reporting and other sources of bias [26].

\section{Results}

\subsection{Study Selection and Characteristics}

The initial literature search resulted in 329 potentially eligible articles. 128 publications were obtained using PubMed, 53 using Scopus and 148 using Web of Science. Additionally, 2 papers were identified through ResearchGate network and were also included. After reading the title and the abstract, 248 records were excluded and the remaining publications $(n=83)$ were checked in full-text. 32 of them were excluded after determining that they did not meet the inclusion criteria, 29 duplicates were removed and finally, a total of 22 studies were included in the present systematic review. The publications dated from 2005 to 2019 and were published in 19 different journals. The flow chart of the selected articles is shown in Figure 1.

The main characteristics and results of the 22 included preclinical studies are shown in Table 1. Most of the preclinical studies were performed in rats (11 out of 22;50\%), followed by rabbits ( 9 out of $22 ; 40 \%$ ), mice ( 1 out of $22 ; 5 \%$ ) and guinea pigs ( 1 out of 22 ; $5 \%$ ). Regarding the animal model selected, in most studies OA was surgically induced by anterior cruciate ligament transection (ACLT) and/or medial meniscectomy (MMT) (16 out of 22; 73\%). Two studies in rats [27,28] and the one which included the mice model [29] used intraarticular injections to chemically induce OA. Only two studies, one involving rabbits [30] and another involving rats [31], used physically immobilisation to induce the OA. Lastly, only one study in guinea pigs was included as a spontaneous model of OA [32]. 


\section{Literature search from 2000 to 2021}
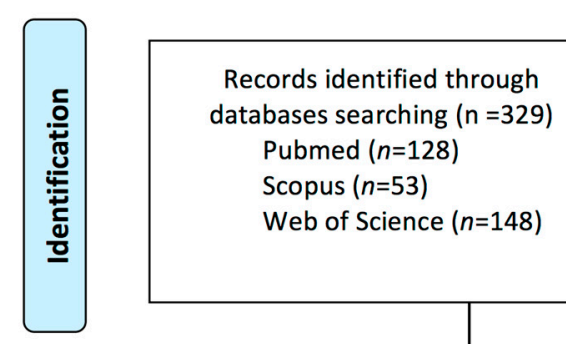

databases searching $(n=329)$

Pubmed $(n=128)$

Scopus $(n=53)$

Web of Science $(n=148)$
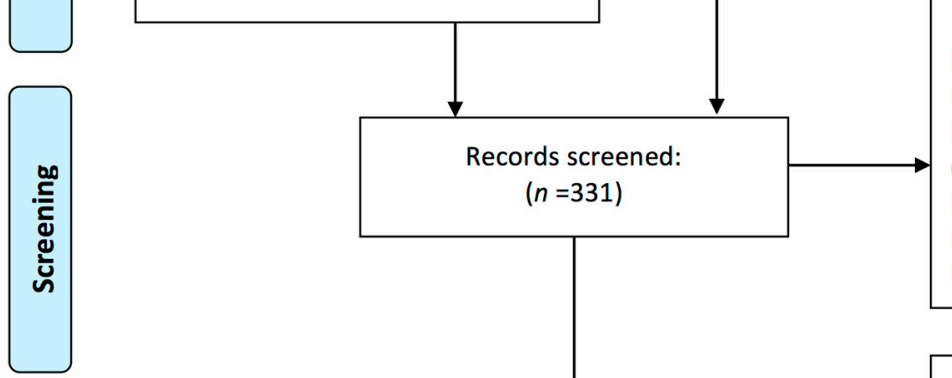

Additional records identified through other sources $(n=2)$

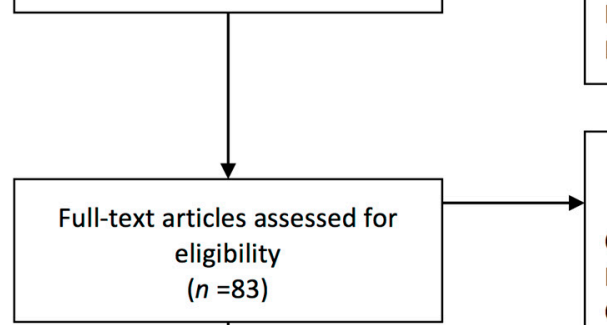

Not inclusion criteria: $(n=108)$

Full-text articles excluded

$$
(n=32)
$$

Other joint: $(n=2)$

Intraarticular: $(n=5)$

Clinical outcomes: $(n=3)$

Not OA model: $(n=13)$

Other: $(n=9)$

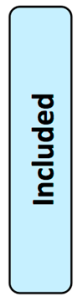

Duplicates removed $(n=29)$

Figure 1. Search strategy according to Preferred Reporting Items for Systematic Reviews and Meta-Analyses (PRISMA) guidelines of preclinical animal studies of the effect of glucosamine and chondroitin sulfate in knee osteoarthritis.

\subsection{Synthesis of the Main Outcomes of the Effect of Glucosamine and/or Chondroitin Sulfate}

The analysed studies were classified based on the therapy evaluated and its effects on the synovial joint tissues, osteophyte development and biochemical markers (Table 2). In the 22 studies included in this review, 26 nutraceutical effects have been evaluated, distributed as follows: Glucosamine sulfate (GS) $(n=6)$, glucosamine hydrochloride $(\mathrm{GH})$ $(n=8)$, chondroitin sulfate (CS) $(n=5), \mathrm{CS}+\mathrm{GH}(n=3)$ and CS+GS $(n=4)$. 
Table 1. Main characteristics and results of preclinical animal studies of the effect of glucosamine and chondroitin sulfate in knee osteoarthritis.

\begin{tabular}{|c|c|c|c|c|c|c|}
\hline References & $\begin{array}{l}\text { Animal Model } \\
(n)\end{array}$ & OA Model & $\begin{array}{l}\text { Therapy: Dosage, Frequency } \\
\text { and Administration Route } \\
\text { (Duration Treatment) }\end{array}$ & Start Point ${ }^{(1)}$ & $\begin{array}{l}\text { Follow-Up }{ }^{(2)} \\
\text { Outcome Measures }\end{array}$ & Main Results \\
\hline $\begin{array}{l}\text { Abdul-Kadir et al. } \\
2019 \text { [33] }\end{array}$ & $\begin{array}{l}\text { New Zealand Rabbit } \\
\text { Male } \\
7-8 \text { months old } \\
\text { (33) }\end{array}$ & $\begin{array}{l}\text { Surgically induced } \\
\text { OA: ACLT }\end{array}$ & $\begin{array}{l}\text { GS }(77.5 \mathrm{mg} / \mathrm{kg} / \text { day) orally } \\
\text { Channa }(51.4 \mathrm{mg} / \mathrm{kg} / \text { day) orally } \\
\text { (8 weeks) }\end{array}$ & 3 weeks & $\begin{array}{l}8 \text { weeks } \\
\text { Macroscopic evaluation. } \\
\text { Histology of cartilage (modified } \\
\text { OARSI score). Histomorphometry } \\
\text { (cartilage roughness and Cg.Th). } \\
\text { Serum biomarkers (COMP, COX-2 } \\
\text { and } \mathrm{PGE}_{2} \text { ) }\end{array}$ & $\begin{array}{l}\text { Both treatment groups showed } \\
\text { lower histopathology changes } \\
\text { compared to the untreated group. } \\
\text { However, Channa showed less } \\
\text { cartilage roughness compared to } \\
\text { GS treated-animals. Channa and } \\
\text { GS significantly } \downarrow \text { COMP levels }\end{array}$ \\
\hline $\begin{array}{l}\text { Jeong et al. } \\
2017 \text { [34] }\end{array}$ & $\begin{array}{l}\text { New Zealand Rabbit } \\
9 \text { month old } \\
(24)\end{array}$ & $\begin{array}{l}\text { Surgically induced } \\
\text { OA: ACLT }\end{array}$ & $\begin{array}{l}\mathrm{GH}(100 \mathrm{mg} / \mathrm{kg} / \text { day }) \text {, orally } \\
\text { Celecoxib }(10 \mathrm{mg} / \mathrm{kg} / \text { day }) \text {, } \\
\text { orally } \\
\text { MucoP }(100 \mathrm{mg} / \mathrm{kg} / \text { day }) \text {, orally } \\
\text { (8 weeks) }\end{array}$ & 3 days & $\begin{array}{l}8 \text { weeks } \\
\text { X-ray. Macroscopic evaluation. } \\
\text { Histology of cartilage. GAG } \\
\text { quantification. TUNEL assay } \\
\text { (Apoptosis of chondrocytes). }\end{array}$ & $\begin{array}{l}\text { Macroscopically GH and MucoP } \\
\text { groups had significantly milder } \\
\text { cartilage damage and fibrillation. } \\
\text { All the treatments investigated } \\
\text { showed significantly reduced } \\
\text { histology degenerative changes } \\
\text { and prevented the apoptosis od } \\
\text { chondrocytes. }\end{array}$ \\
\hline $\begin{array}{l}\text { Permuy et al. } 2015 \\
\text { [36] }\end{array}$ & $\begin{array}{l}\text { New Zealand Rabbit } \\
\text { Female } \\
\text { Adult } \\
(56)\end{array}$ & $\begin{array}{l}\text { Surgically induced } \\
\text { OA: ACLT and } \\
\text { partial MMT }\end{array}$ & $\begin{array}{l}\text { GS }(21.5 \mathrm{mg} / \mathrm{kg} / \text { day) orally } \\
\text { CS ( } 11.5 \mathrm{mg} / \mathrm{kg} / \text { day) i.p. } \\
\text { HA }(0.3 \mathrm{mg} / \mathrm{kg} / \text { week }) \text { i.a. } \\
\text { DC }(1.5 \mathrm{mg} / \mathrm{kg} / \text { day) orally } \\
\text { RIS ( } 0.07 \mathrm{mg} / \mathrm{kg} / \text { day) orally } \\
\text { GS + RIS daily orally } \\
\text { (8 weeks) }\end{array}$ & 3 weeks & $\begin{array}{l}11 \text { weeks } \\
\text { Histology of cartilage and } \\
\text { synovial membrane (OARSI } \\
\text { score). } \\
\text { Histomorphometric evaluation } \\
\text { (Tb.A, Tb.Sp, SB.Th, FI, Cg.Th) } \\
\text { and } \mu-C T \text { (vBMD, BV /TV, Tb.Th, } \\
\text { Tb.Sp, Tb.N, Tb.Pf, SMI, nCgTh) }\end{array}$ & $\begin{array}{l}\text { GS and CS prevented cartilage } \\
\text { swelling but did not reduce the } \\
\text { cartilage damage or the } \\
\text { superficial fibrillation. In } \\
\text { addition, they did not have any } \\
\text { effect on synovial and } \\
\text { subchondral bone. }\end{array}$ \\
\hline
\end{tabular}


Table 1. Cont.

\begin{tabular}{|c|c|c|c|c|c|c|}
\hline References & $\begin{array}{l}\text { Animal Model } \\
(n)\end{array}$ & OA Model & $\begin{array}{l}\text { Therapy: Dosage, Frequency } \\
\text { and Administration Route } \\
\text { (Duration Treatment) }\end{array}$ & Start Point & $\begin{array}{l}\text { Follow-Up }{ }^{(2)} \\
\text { Outcome Measures }\end{array}$ & Main Results \\
\hline $\begin{array}{l}\text { Ohnishi et al. } 2013 \\
\text { [37] }\end{array}$ & $\begin{array}{l}\text { Japanese Rabbit } \\
\text { Female } \\
12 \text { weeks old } \\
(12)\end{array}$ & $\begin{array}{l}\text { Surgically induced } \\
\text { OA: ACLT }\end{array}$ & $\begin{array}{l}\text { FCP (1000 mg/day), orally } \\
\text { GH (1000 mg/day), orally } \\
\text { FCP + GS daily orally } \\
\text { (4 weeks) }\end{array}$ & 0 days & $\begin{array}{l}4 \text { weeks } \\
\text { Macroscopic evaluation. } \\
\text { Histology of cartilage (modified } \\
\text { Mankin score). IHQ (CTX-II). } \\
\text { Serum biomarkers (CS-846, HA, } \\
\text { KS) }\end{array}$ & $\begin{array}{l}\text { Administration of FCP and/or } \\
\text { GH showed chondroprotective } \\
\text { effects. However, there were no } \\
\text { significant differences in the } \\
\text { biomarker concentrations } \\
\text { comparing with untreated } \\
\text { animals ( } \uparrow C S 846 \text { and HA) }\end{array}$ \\
\hline $\begin{array}{l}\text { Wang et al. } \\
2007 \text { [38] }\end{array}$ & $\begin{array}{l}\text { New Zealand Rabbit } \\
\text { Male } \\
9 \text { month old } \\
(26)\end{array}$ & $\begin{array}{l}\text { Surgically induced } \\
\text { OA: ACLT }\end{array}$ & $\begin{array}{l}\text { GH }(100 \mathrm{mg} / \text { day) orally } \\
\text { (8 weeks) }\end{array}$ & 1 day & $\begin{array}{l}8 \text { weeks } \\
\text { Macroscopic evaluation. X-ray } \\
\text { absorptiometry scanner } \\
\text { (BMD). Histomorphomethry } \\
\text { (osteoid volumen, osteoid } \\
\text { thicknes, BV/TV, Tb.Th, Tb.Sp, } \\
\text { Tb.N, SB.Th) }\end{array}$ & $\begin{array}{l}\text { GH tended to have lower } \\
\text { severity of cartilage lesions but } \\
\text { this difference was not significant. } \\
\text { Additionally, GH prevented the } \\
\text { subchondral bone changes and } \\
\text { prevented the trabecular bone } \\
\text { loss. }\end{array}$ \\
\hline $\begin{array}{l}\text { Kobayashi et al. } \\
2005 \text { [39] }\end{array}$ & $\begin{array}{l}\text { Japanese Rabbit } \\
\text { Male } \\
13 \text { weeks old } \\
(30)\end{array}$ & $\begin{array}{l}\text { Surgically induced } \\
\text { OA: } \\
\text { Partial MMT }\end{array}$ & $\begin{array}{l}\mathrm{GH}(1000 \mathrm{mg} / \mathrm{kg} / \text { day })+\mathrm{CS}(800 \\
\mathrm{mg} / \mathrm{kg} / \text { day }) \text {, orally } \\
\mathrm{GH}+\mathrm{CS}+\text { Fursultiamine }(100 \\
\mathrm{mg} / \mathrm{kg} / \text { day }) \text {, orally } \\
\text { (8 weeks) }\end{array}$ & 3 days & $\begin{array}{l}8 \text { weeks } \\
\text { Macroscopic evaluation. } \\
\text { Histology of cartilage (modified } \\
\text { Mankin score). IHQ (MMP-1) }\end{array}$ & $\begin{array}{l}\text { The GH + CS treatment slightly } \\
\text { reduce the severity of cartilage } \\
\text { lesions but no significantly. Only } \\
\text { the group with received the } \\
\text { combined treatment with } \\
\text { fursultiamine showed a } \\
\text { significant chondroprotective } \\
\text { effects and } \downarrow M M P-1 \text {. }\end{array}$ \\
\hline $\begin{array}{l}\text { Tiraloche et al. } \\
2005 \text { [40] }\end{array}$ & $\begin{array}{l}\text { New Zealand Rabbit } \\
\text { Male } \\
9 \text { month old } \\
\text { (39) }\end{array}$ & $\begin{array}{l}\text { Surgically induced } \\
\text { OA: ACLT }\end{array}$ & $\begin{array}{l}\text { GH }(100 \mathrm{mg} / \text { day) orally } \\
\text { (8 weeks) }\end{array}$ & 3 weeks & $\begin{array}{l}11 \text { weeks } \\
\text { Macroscopic evaluation. } \\
\text { Histology of cartilage (modified } \\
\text { Mankin score). GAG } \\
\text { quantification and total type II } \\
\text { collagen. }\end{array}$ & $\begin{array}{l}\text { GH administration tended to } \\
\text { have lower macroscopic severity } \\
\text { grades compared with placebo } \\
\text { group, but it was not significant. } \\
\text { Regarding histology parameters, } \\
\text { GH did not prevent the cartilage } \\
\text { erosion nor superficial } \\
\text { fibrillation. }\end{array}$ \\
\hline
\end{tabular}


Table 1. Cont.

\begin{tabular}{|c|c|c|c|c|c|c|}
\hline References & $\begin{array}{l}\text { Animal Model } \\
(n)\end{array}$ & OA Model & $\begin{array}{l}\text { Therapy: Dosage, Frequency } \\
\text { and Administration Route } \\
\text { (Duration Treatment) }\end{array}$ & Start Point ${ }^{(1)}$ & $\begin{array}{l}\text { Follow-Up }{ }^{(2)} \\
\text { Outcome Measures }\end{array}$ & Main Results \\
\hline $\begin{array}{l}\text { Torelli et al. } 2005 \\
\text { [30] }\end{array}$ & $\begin{array}{l}\text { Norflok Rabbit } \\
\text { Female } \\
2.5-3 \text { monts old } \\
(40)\end{array}$ & $\begin{array}{l}\text { Physically induced } \\
\text { OA: Immobilization } \\
\text { (12 weeks) }\end{array}$ & $\begin{array}{l}\text { CS (120 mg/week) s.c. } \\
\text { (12 weeks) }\end{array}$ & 0 days & $\begin{array}{l}12 \text { weeks } \\
\text { Histology of cartilage } \\
\text { (hematoxylin-eosin, Masson } \\
\text { trichrome and picrosirius red) and } \\
\text { proteoglycan content. }\end{array}$ & $\begin{array}{l}\text { CS did not reduce the } \\
\text { histological changes such as } \\
\text { cartilage fibrillation, chondrocyte } \\
\text { disorganization and } \\
\downarrow \text { proteoglycan, compared to } \\
\text { untreated animals. }\end{array}$ \\
\hline Sun et al. 2018 [27] & $\begin{array}{l}\text { Sprague Dawley Rat } \\
\text { Male } \\
5-6 \text { weeks old } \\
(48)\end{array}$ & $\begin{array}{l}\text { Chemically } \\
\text { induced OA: } \\
\text { MIA }\end{array}$ & $\begin{array}{l}\text { CSSB ( } 50 \mathrm{mg} / \mathrm{kg} / \text { day }) \text {, orally } \\
\text { CSSB }(100 \mathrm{mg} / \mathrm{kg} / \text { day), orally } \\
\text { CSSB }(200 \mathrm{mg} / \mathrm{kg} / \text { day }) \text {, orally } \\
\text { CS }(200 \mathrm{mg} / \mathrm{kg} / \text { day }) \text {, orally } \\
(1 \mathrm{month})\end{array}$ & 0 days & $\begin{array}{l}\text { In-vivo paint and bearing test, } \\
\text { daily } \\
1 \text { month } \\
\text { Histology of cartilage (OARSI } \\
\text { score). Synovial markers (IL-1 } \beta \text {, } \\
\text { TNF-alpha, PGE } \text { Pand NO). }_{\text {and }} \text { Western-blot (MMP-1, MMP-3 } \\
\text { and TIMP-1) }\end{array}$ & $\begin{array}{l}\text { Treated groups exhibited } \\
\text { significant reduced } \\
\text { histopathological cartilage } \\
\text { changes, relieved joint pain and } \\
\text { showed } \downarrow \text { IL1 } \beta \text {, TNF-alpha, } \text { PGE }_{2} \\
\text { and NO. Additionally, regulated } \\
\text { the protein expression }(\downarrow M M P-1 \\
\text { and MMP-3 and } \uparrow \text { TIMP-1). } \\
\text { Dose-dependent manner. }\end{array}$ \\
\hline $\begin{array}{l}\text { Wang et al. } 2018 \\
\text { [41] }\end{array}$ & $\begin{array}{l}\text { Sprague Dawley Rat } \\
(40)\end{array}$ & $\begin{array}{l}\text { Surgically induced } \\
\text { OA: ACLT and } \\
\text { MMT }\end{array}$ & $\begin{array}{l}\text { GS }(2,5 \text { or } 10 \mathrm{mg} / \mathrm{kg} / \text { day }) \text {, } \\
\text { intraperitonally } \\
(1 \text { month })\end{array}$ & 0 days & $\begin{array}{l}1 \text { month } \\
\text { Histology of cartilage. IHQ (type } \\
\text { II collagen). Synovial fluid } \\
\text { inflammatory mediators (NO and } \\
\text { IL1 } \beta \text { ). Western-blot (MMP-1, } \\
\text { MMP-13). qPCR (TIMP-1) }\end{array}$ & $\begin{array}{l}\text { Glucosamine treatment } \\
\text { prevented cartilage degradation, } \\
\text { up-regulated the levels of type II } \\
\text { collagen and } \downarrow \text { MMP- } 1 \text { and } \\
\text { MMP-13 and } \uparrow \text { TIMP- } 1 \text {, in a } \\
\text { dose-dependent manner. }\end{array}$ \\
\hline
\end{tabular}


Table 1. Cont.

\begin{tabular}{|c|c|c|c|c|c|c|}
\hline References & $\begin{array}{l}\text { Animal Model } \\
(n)\end{array}$ & OA Model & $\begin{array}{l}\text { Therapy: Dosage, Frequency } \\
\text { and Administration Route } \\
\text { (Duration Treatment) }\end{array}$ & Start Point ${ }^{(1)}$ & $\begin{array}{l}\text { Follow-Up }{ }^{(2)} \\
\text { Outcome Measures }\end{array}$ & Main Results \\
\hline Ren et al. 2017 [42] & $\begin{array}{l}\text { Rat } \\
\text { Male } \\
(24)\end{array}$ & $\begin{array}{l}\text { Surgically induced } \\
\text { OA: ACLT }\end{array}$ & $\begin{array}{l}\text { CSSM ( } 25 \mathrm{mg} / \text { twice daily), orally } \\
\text { CS (shark) ( } 25 \mathrm{mg} / \text { twice daily), } \\
\text { orally } \\
\text { ( } 6 \text { weeks) }\end{array}$ & 4 weeks & $\begin{array}{l}10 \text { weeks } \\
\text { Macroscopic evaluation. } \\
\text { Histology of cartilage (Mankin } \\
\text { score). Synovial fluid } \\
\text { inflammatory mediators (IL-1 } \beta \text {, } \\
\text { TNF-alpha, PGE } 2 \text { ). TUNNEL } \\
\text { assay (Apoptosis of chondrocytes). } \\
\text { Western-blot (MMP-1 and } \\
\text { TIMP-1). }\end{array}$ & $\begin{array}{l}\text { Treated groups showed } \\
\text { chondroprotective effects by } \\
\text { inhibiting the cartilage } \\
\text { degradation and the apoptosis of } \\
\text { chondrocytes. } \downarrow \text { IL- } 1 \beta \text {, } \\
\text { TNF-alpha, PGE } 2, \downarrow \text { MMP- } 1 \text { and } \\
\uparrow \text { TIMP- } 1 \text {. }\end{array}$ \\
\hline $\begin{array}{l}\text { Sanches et al. } 2017 \\
\text { [43] }\end{array}$ & $\begin{array}{l}\text { Wistar Rat } \\
\text { Male } \\
8 \text { weeks old } \\
(40)\end{array}$ & $\begin{array}{l}\text { Surgically induced } \\
\text { OA: ACLT }\end{array}$ & $\begin{array}{l}\mathrm{CS}(400 \mathrm{mg} / \mathrm{kg})+\mathrm{GS}(500 \\
\mathrm{mg} / \mathrm{kg}) 3 \text { days } / \text { week, orally } \\
\mathrm{CS}+\mathrm{GS}+\text { photobiomodulation } \\
(29 \text { days })\end{array}$ & 2 days & $\begin{array}{l}30 \text { days } \\
\text { Histology of cartilage (OARSI } \\
\text { score). Histomorphometry } \\
\text { (chondrocytes density and CgTh). } \\
\text { IHQ (IL-1 } \beta \text {, IL-10, type II } \\
\text { collagen) }\end{array}$ & $\begin{array}{l}\text { All treated groups showed lower } \\
\text { degenerative histological } \\
\text { changes and chondrocytes } \\
\text { density. Animals treated with CS } \\
+ \text { GS + PBM showed significant } \\
\downarrow \text { IL-1 } \beta \text { and } \uparrow I L-10 \text { and type II } \\
\text { collagen compared to untreated } \\
\text { group. }\end{array}$ \\
\hline $\begin{array}{l}\text { Terencio et al. } \\
2016 \text { [44] }\end{array}$ & $\begin{array}{l}\text { Wistar Rat } \\
\text { Female } \\
10 \text { weeks old } \\
(45)\end{array}$ & $\begin{array}{l}\text { OVX }+ \\
\text { Surgically induced } \\
\text { OA: } \\
\text { ACLT ( } 2 \text { weeks } \\
\text { post-OVX) }\end{array}$ & $\begin{array}{l}\mathrm{CS}(140 \mathrm{mg} / \mathrm{kg} / \text { day })+\mathrm{GH}(175 \\
\mathrm{mg} / \mathrm{kg} / \text { day }), \text { orally } \\
(12 \text { weeks })\end{array}$ & -2 weeks & $\begin{array}{l}10 \text { weeks } \\
\text { Histology of cartilage and } \\
\text { synovial membrane (OARSI } \\
\text { score). Synovial fluid } \\
\text { inflammatory mediators by ELISA } \\
\text { (IL-1 } \beta \text {, TNF-alpha) and } \\
\text { radioimmunoassay (PGE } 2 \text { ). } \\
\text { Serum biomarkers (CTX-II, } \\
\text { MMP-3, OPG, RANKL and } \\
\text { osteocalcin). } \mu \text {-CT (BV/TV, Tb.Th, } \\
\text { Tb.N and vBMD) }\end{array}$ & $\begin{array}{l}\text { OARSI scores of cartilage } \\
\text { degradation were decreased in } \\
\text { the treated group. CS and GH } \\
\text { showed reduced levels of } \\
\text { inflammatory mediators ( } \downarrow \text { IL- } 1 \beta \\
\text { and TNF-alpha, CTX-II, MMP-3, } \\
\text { OPG, RANKL) and a tendency to } \\
\text { prevent the bone microstructural } \\
\text { changes ( } \uparrow \text { vMD) although, } \\
\text { without statistical significance. }\end{array}$ \\
\hline
\end{tabular}


Table 1. Cont.

\begin{tabular}{|c|c|c|c|c|c|c|}
\hline References & $\begin{array}{l}\text { Animal Model } \\
(n)\end{array}$ & OA Model & $\begin{array}{l}\text { Therapy: Dosage, Frequency } \\
\text { and Administration Route } \\
\text { (Duration Treatment) }\end{array}$ & Start Point & $\begin{array}{l}\text { Follow-Up }{ }^{(2)} \\
\text { Outcome Measures }\end{array}$ & Main Results \\
\hline $\begin{array}{l}\text { Panahafir et al. } \\
2014 \text { [45] }\end{array}$ & $\begin{array}{l}\text { Sprague Dawley Rat } \\
9 \text { month old } \\
(27)\end{array}$ & $\begin{array}{l}\text { Surgically } \\
\text { induced OA: } \\
\text { KTI }\end{array}$ & $\begin{array}{l}\text { Celecoxib }(2.86 \mathrm{mg} / \mathrm{kg} / \text { day }) \text {, } \\
\text { orally } \\
\text { GH }(192 \mathrm{mg} / \mathrm{kg} / \text { day }) \text {, orally } \\
(4,8 \text { or } 12 \text { weeks })\end{array}$ & 0 days & $\begin{array}{l}4,8 \text { or } 12 \text { weeks } \\
\mu \text {-CT and MRI. Histology of } \\
\text { cartilage and synovial membrane } \\
\text { (RAKSS score) }\end{array}$ & $\begin{array}{l}\text { None of the treatments } \\
\text { prevented cartilage loss, synovial } \\
\text { inflammation or subchondral } \\
\text { sclerosis. Additionally, GH failed } \\
\text { to prevent the osteophyte } \\
\text { formation }\end{array}$ \\
\hline $\begin{array}{l}\text { Wen et al. } 2010 \\
\text { [46] }\end{array}$ & $\begin{array}{l}\text { Wistar Rat } \\
\text { Male } \\
2 \text { moth old } \\
(36)\end{array}$ & $\begin{array}{l}\text { Surgically induced } \\
\text { OA: ACLT }\end{array}$ & $\begin{array}{l}\text { GS }(250 \mathrm{mg} / \mathrm{kg} / \text { day } / \text {, orally } \\
\text { (10 weeks) }\end{array}$ & 5 weeks & $\begin{array}{l}\text { 3, 6, 9, 12, } 15 \text { and } 18 \text { weeks } \\
\text { Allodynia and weight-bearing } \\
\text { Macroscopic evaluation. } \\
\text { Histology of cartilage and } \\
\text { synovial membrane (Mankin } \\
\text { score). IHQ (p38, JNK, ERK and } \\
\text { MAPKs) }\end{array}$ & $\begin{array}{l}\text { GS treated animals showed } \\
\text { significantly lower cartilage } \\
\text { damage and suppressed the } \\
\text { synovial inflammation. } \\
\text { Additionally, reduced the } \\
\text { allodynia and weight bearing. } \\
\downarrow \text { p38 and JNK, } \uparrow \text { ERK. }\end{array}$ \\
\hline $\begin{array}{l}\text { Naito et al. } 2010 \\
\text { [47] }\end{array}$ & $\begin{array}{l}\text { Sprague Dawley Rat } \\
\text { Male } \\
10 \text { week old } \\
(18)\end{array}$ & $\begin{array}{l}\text { Surgically induced } \\
\text { OA: ACLT }\end{array}$ & $\begin{array}{l}\mathrm{GH}(1000 \mathrm{mg} / \mathrm{kg} / \text { day }) \text {, orally } \\
\text { (8 weeks) }\end{array}$ & 0 days & $\begin{array}{l}56 \text { days } \\
\text { Macroscopic evaluation. } \\
\text { Histology of cartilage (Mankin } \\
\text { score). Serum biomarkers (CTX-I, } \\
\text { CTX-II, CPII) }\end{array}$ & $\begin{array}{l}\text { GH administration suppressed } \\
\text { the macroscopic changes and } \\
\text { reduced the Mankin scores, but } \\
\text { not significantly. } \downarrow \text { CTX-II, } \uparrow \text { CPII }\end{array}$ \\
\hline $\begin{array}{l}\text { Silva et al. } 2009 \\
\text { [48] }\end{array}$ & $\begin{array}{l}\text { Wistar Rat } \\
\text { Male } \\
(?)\end{array}$ & $\begin{array}{l}\text { Surgically induced } \\
\text { OA: ACLT }\end{array}$ & $\begin{array}{l}\text { GS }(500 \mathrm{mg} / \mathrm{kg} / \text { day }), \text { orally } \\
\mathrm{GS}(500 \mathrm{mg} / \mathrm{kg} / \text { day })+\mathrm{CS}(400 \\
\mathrm{mg} / \mathrm{kg} / \text { day }), \text { orally } \\
\text { (70 days) }\end{array}$ & -7 days & $\begin{array}{l}\text { In-vivo joint pain } \\
70 \text { days } \\
\text { Histology of cartilage (OARSI } \\
\text { score). Densitometry (CS content } \\
\text { of cartilage) }\end{array}$ & $\begin{array}{l}\text { GS + CS significantly prevented } \\
\text { the cartilage histology alterations. } \\
\text { Additionally, significantly } \\
\text { reversed the increase in the CS } \\
\text { cartilage quantification and } \\
\text { reduced the joint pain. }\end{array}$ \\
\hline
\end{tabular}


Table 1. Cont.

\begin{tabular}{|c|c|c|c|c|c|c|}
\hline References & $\begin{array}{l}\text { Animal Model } \\
(n)\end{array}$ & OA Model & $\begin{array}{l}\text { Therapy: Dosage, Frequency } \\
\text { and Administration Route } \\
\text { (Duration Treatment) }\end{array}$ & Start Point ${ }^{(1)}$ & $\begin{array}{l}\text { Follow-Up }{ }^{(2)} \\
\text { Outcome Measures }\end{array}$ & Main Results \\
\hline $\begin{array}{l}\text { Ivanovska et al. } \\
2011 \text { [29] }\end{array}$ & $\begin{array}{l}\text { Outbred ICR (CD-2) } \\
\text { Mice } \\
\text { Male } \\
\text { 10-12 weeks old } \\
(50)\end{array}$ & $\begin{array}{l}\text { Chemically } \\
\text { induced OA: } \\
\text { CIOA }\end{array}$ & $\begin{array}{l}\text { GS }(20 \mathrm{mg} / \mathrm{kg} / \text { day }) \text {, orally } \\
\text { GH }(20 \mathrm{mg} / \mathrm{kg} / \text { day }) \text {, orally } \\
\text { (20 days) }\end{array}$ & 0 or 7 days & $\begin{array}{l}30 \text { days } \\
\text { Histology of cartilage. Osteophyte } \\
\text { area. Synovial fluid inflammatory } \\
\text { mediators (RANKL, TNF-alpha, } \\
\text { IL-6, IL-4 and IL-10). IHQ } \\
\text { (RANKL, BMP-2) }\end{array}$ & $\begin{array}{l}\text { GH significantly reduces the } \\
\text { cartilage damage and osteophyte } \\
\text { area. Additionally, ameliorates } \\
\text { the OA progression by regulating } \\
\text { the degree of bone resorption } \\
\text { and bone remodeling. } \downarrow \text { RANKL, } \\
\text { BMP-2 and IL-6, } \uparrow \text { IL-10. }\end{array}$ \\
\hline $\begin{array}{l}\text { Taniguchi et al. } \\
2011 \text { [32] }\end{array}$ & $\begin{array}{l}\text { Hartley Guinea-pig } \\
\text { Female } \\
3 \text { weeks old } \\
(50)\end{array}$ & $\begin{array}{l}\text { Spontaneusly } \\
\text { model: } \\
\text { Naturally } \\
\text { occurring }\end{array}$ & $\begin{array}{l}\mathrm{GH}(200 \mathrm{mg} / \mathrm{kg} / \text { day }), \text { orally } \\
\mathrm{CS}(200 \mathrm{mg} / \mathrm{kg} / \text { day }) \text {, orally } \\
(8,12 \text { or } 18 \mathrm{months})\end{array}$ & - & $\begin{array}{l}8,12 \text { or } 18 \text { months } \\
\text { Histology of cartilage (modified } \\
\text { Mankin score). TUNEL assay } \\
\text { (Apoptosis of chondrocytes). } \\
\text { mRNA levels for cartilage tissue } \\
\text { (MMP-3, MMP-8, MMP-13, } \\
\text { collagen type II and aggrecan) }\end{array}$ & $\begin{array}{l}\text { Long-term GH or CS } \\
\text { administration reduced the } \\
\text { cartilage degeneration. } \\
\text { Additionally, inhibited the loss of } \\
\text { cartilage total RNA and the } \\
\text { increase of MMP-3 mRNA }\end{array}$ \\
\hline
\end{tabular}

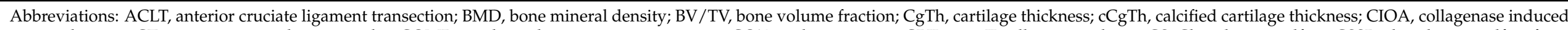

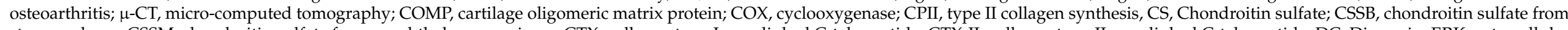

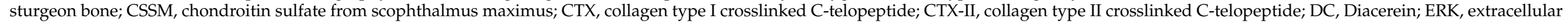

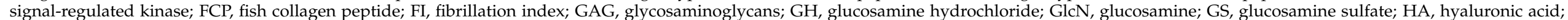

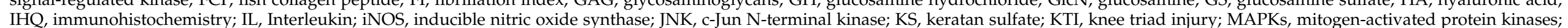

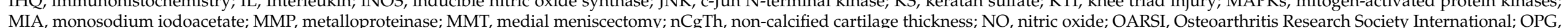

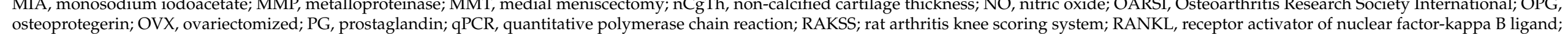

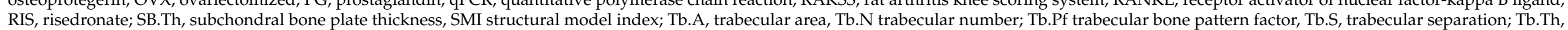

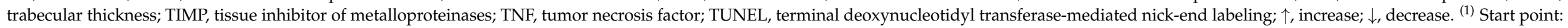
time between induced OA and treatment administration. ${ }^{(2)}$ Follow-Up: time between induced OA and the evaluations carried out in the study. 
Table 2. Synthesis of main outcomes of the effect of nutraceuticals.

\begin{tabular}{|c|c|c|c|c|c|c|c|}
\hline Nutraceutical & Reference & Initial Adminst Ration & $\mathrm{C}$ & SB & SM & OST & BM \\
\hline \multirow{6}{*}{$\begin{array}{l}\text { Glucosamine sulfate (GS) } \\
n=6\end{array}$} & Abdul-Kadir et al. [33] & Delayed & + & $x$ & $x$ & $x$ & + \\
\hline & Permuy et al. [36] & Delayed & - & - & - & $x$ & $x$ \\
\hline & Salman et al. [31] & Early & + & $x$ & $x$ & $x$ & + \\
\hline & Wang et al. [41] & Early & + & $\mathrm{x}$ & $x$ & $\mathrm{x}$ & + \\
\hline & Wen et al. [46] & Delayed & + & $x$ & + & $x$ & + \\
\hline & Ivanovska et al. [29] & Early & - & $\mathrm{x}$ & $\mathrm{x}$ & - & - \\
\hline \multirow{8}{*}{$\begin{array}{l}\text { Glucosamine } \\
\text { hydrochloride }(\mathrm{GH}) \\
n=8\end{array}$} & Jeong et al. [34] & Early & + & $\mathrm{x}$ & $\mathrm{x}$ & $x$ & + \\
\hline & Ohnishi et al. [37] & Early & + & $x$ & $x$ & $x$ & - \\
\hline & Wang et al. [38] & Early & $?$ & + & $x$ & $x$ & $x$ \\
\hline & Tiraloche et al. [40] & Delayed & $?$ & $\mathrm{x}$ & $x$ & $x$ & - \\
\hline & Panahafir et al. [45] & Early & - & - & - & - & $x$ \\
\hline & Naito et al. [47] & Early & $?$ & $\mathrm{x}$ & $\mathrm{x}$ & $\mathrm{x}$ & + \\
\hline & Ivanovska et al. [29] & Early & + & $x$ & $x$ & + & + \\
\hline & Taniguchi et al. [32] & Pre-emptive & + & $x$ & $x$ & $x$ & + \\
\hline \multirow{5}{*}{$\begin{array}{l}\text { Chondroitin sulfate } \\
n=5\end{array}$} & Permuy et al. [36] & Delayed & - & - & - & $x$ & $x$ \\
\hline & Torelli et al. [30] & Early & - & $x$ & $x$ & $x$ & $x$ \\
\hline & Sun et al. [27] & Early & + & $\mathrm{x}$ & $\mathrm{x}$ & $\mathrm{x}$ & + \\
\hline & Ren et al. [42] & Delayed & + & $x$ & $x$ & $x$ & + \\
\hline & Taniguchi et al. [32] & Pre-emptive & + & $\mathrm{x}$ & $x$ & $\mathrm{x}$ & + \\
\hline \multirow{3}{*}{$\begin{array}{l}\text { Chondroitin sulfate }+\mathrm{GH} \\
n=3\end{array}$} & Roman-Blas et al. [35] & Pre-emptive & - & - & - & $\mathrm{x}$ & - \\
\hline & Kobayashi et al. [39] & Early & $?$ & $x$ & $x$ & $x$ & $?$ \\
\hline & Terencio et al. [44] & Pre-emptive & + & $?$ & $?$ & $x$ & + \\
\hline \multirow{4}{*}{$\begin{array}{l}\text { Chondroitin sulfate }+ \text { GS } \\
n=4\end{array}$} & Roman-Blas et al. [35] & Pre-emptive & - & - & - & $x$ & - \\
\hline & Sanches et al. [43] & Early & + & $\mathrm{x}$ & $x$ & $x$ & $?$ \\
\hline & Lee et al. [28] & Early & $x$ & + & $x$ & $x$ & $\mathrm{x}$ \\
\hline & Silva et al. [48] & Pre-emptive & + & $\mathrm{x}$ & $\mathrm{x}$ & $x x$ & + \\
\hline
\end{tabular}

C cartilage, SB subchondral bone, SM synovial membrane, OST osteophyte, BM biochemical markers. (+) Positive effect; (-) negative effect or no effect; (?) unclear or not significantly effect; (x) not included. Therapy initial administration: Pre-emptive (before OA induction), early (OA induction- 14 days post); delayed ( $>14$ days post-OA induction).

Most of the publications analysed the glucosamine effect $(n=14)$ in its hydrochloride $(n=8)$ or sulfate $(n=6)$ form. Less number of publications analysed the effect of the chondroitin sulfate, administered either alone $(n=5)$ or in combination with glucosamine sulfate $(n=4)$ or glucosamine hydrochloride $(n=3)$. Additionally, it should be noted that one study included the evaluation of glucosamine sulfate and chondroitin sulfate separately [36], another the glucosamine sulfate and the glucosamine hydrochloride [29], another the effect of the glucosamine hydrochloride and the chondroitin sulfate [32] and another the combination of chondroitin sulfate plus glucosamine hydrochloride against chondroitin sulfate plus glucosamine sulfate [35]. Consequently, as we explained before, within the 22 studies included in the present systematic review, 26 evaluations of the nutraceutical effect alone or in combination were carried out.

Regarding the parameters evaluated, the cartilage response is by far the most assessed, being included in 25 evaluations out of 26. Positive chondroprotective effects were identified in approximately half of the evaluations (14 out of $25 ; 56 \%$ ). In the individual analyses, the results were as follows: glucosamine sulfate (4 out of $6 ; 67 \%)$, glucosamine hydrochloride (4 out of $8 ; 50 \%$ ), chondroitin sulfate ( 3 out of $5 ; 60 \%$ ); chondroitin sulfate plus glucosamine hydrochloride (1 out of $3 ; 33 \%$ ) and chondroitin sulfate plus glucosamine sulfate ( 2 out of $3 ; 67 \%$ ). The biochemical markers of OA were the second most studied parameter in this systematic review and was included in 20 out of 26 therapy assessments. Nutraceuticals showed a positive effect in 13 of them (13 out of 20; 65\%). Specifically, in terms of glucosamine therapies, we identified positive responses in 4 out of $5(80 \%)$ 
sulfate formulations and in 4 out of $6(67 \%)$ hydrochloride ones. With respect to chondroitin sulfate, 3 out of 5 publications included biomarker evaluations and, in this case, all of them showed fewer biochemical alterations in the treated groups. The subchondral bone changes were determined in 8 out of the total number of included evaluations, identifying beneficial effects in only two of the publications ( 2 out of $8 ; 25 \%$ ) $[28,38]$. The synovial inflammation was evaluated in 7 studies, showing supressed synovitis in only one of them (1 out of 7 ; 14\%) [46]. Finally, the osteophyte development was evaluated in 3 studies, but only in one of them a reduced osteophyte formation was observed after glucosamine hydrochloride administration [29].

\subsection{Therapy Duration and Initial Administration at Baseline}

The nutraceutical therapy periods were shown in Table 3 . The majority of the preclinical studies included in the systematic review were based on short-term therapies $(n=15)$. Intermediate-term therapies were employed in 6 of the selected publications, with nutraceutical treatment periods lasting 8-24 weeks. Lastly, the review only included a publication which studied the long-term therapy response in a guinea pig spontaneous OA model [32].

Regarding the therapy timing initiation in relation to OA induction, most of the studies applied early therapy administrations, up to only 14 days post experimental OA induction. 5 out of 22 articles studied the effect of these therapies in delayed administrations ( $>14$ days post OA induction) and finally, 4 studies focused on the pre-emptive responses (before OA induction) (Table 2). The chondroprotective effect was observed in 7 out of the 13 publications with early treatment administrations, making up for $54 \%$, 3 out of 5 publications with delayed initial treatments, corresponding to $60 \%$ and finally, 3 out of 4 pre-emptive protocols making up for $75 \%$.

\subsection{Quality and Risk-of-Bias Assessments}

The quality assessments of the preclinical studies based on the essential 10 items of the ARRIVE guidelines were summarised in Figure 2. The individual analysis of the manuscripts showed that at items 4 "Randomisation" and 5 "Blinding", information was not adequately reported in $32 \%$ and $50 \%$ of the studies, respectively. By contrast, at items 1 "Study design", 6 "Outcome measures", 7 "Statistical methods", 8 "Experimental animals" and 10 "Results", adequate and clear information was reported in the experimental studies, with percentages of $91 \%, 77 \%, 68 \%, 86 \%$ and $73 \%$ of the studies. Other items, such as 2 "Sample size", 3 "Inclusion and exclusion criteria" and 9 "Experimental procedures" were graded as unclear with percentages of $82 \%, 68 \%$ and $82 \%$ of the studies, due to partially reported or insufficient experimental details provided in the studies.

Figure 3 summarises the risk-of-bias distribution results obtained with the SYRCLE tool. The lower risk of bias was observed at items 1 "Sequence generation, 7 "Blinding of outcome assessor" and 9 "Selective outcome reporting", with percentages of $59 \%, 55 \%$ and $64 \%$, respectively. The higher risk of bias was assigned at item 3 "Allocation concealment" with a percentage of $68 \%$, whereas high frequencies of unclear risk of bias ratings were assigned at items 4 "Random housing", 5 "Blinding of caregivers", 6 "Random outcome assessment", 8 "Incomplete data outcome" and 10 "Other sources of bias", with percentages of $100 \%, 91 \%, 91 \%, 73 \%$ and $63 \%$, respectively. 
Table 3. Therapy duration of nutraceuticals.

\begin{tabular}{|c|c|c|c|c|c|c|c|c|c|}
\hline \multirow{2}{*}{ Animal Model } & \multicolumn{3}{|c|}{ Short-term ( $\leq 8$ Weeks) } & \multicolumn{3}{|c|}{ Intermediate-Term ( $>8$ to $<24$ Weeks) } & \multicolumn{3}{|c|}{ Long-Term ( $\geq 24$ Weeks) } \\
\hline & Reference & Therapy & Duration & Reference & Therapy & Duration & Reference & Therapy & Duration \\
\hline \multirow[t]{6}{*}{ Rabbit } & Abdul-Kadir et al. [33] & GS & 8 weeks & Roman-Blas et al. [35] & $\mathrm{GH} / \mathrm{GS}+\mathrm{CS}$ & 14 weeks & & & \\
\hline & Jeong et al. [34] & GH & 8 weeks & Torelli et al. [30] & CS & 12 weeks & & & \\
\hline & Ohnishi et al. [37] & GH & 4 weeks & & & & & & \\
\hline & Wang et al. [38] & GH & 8 weeks & & & & & & \\
\hline & Kobayashi et al. [39] & $\mathrm{GH}+\mathrm{CS}$ & 8 weeks & & & & & & \\
\hline & Tiraloche et al. [40] & $\mathrm{GH}$ & 8 weeks & & & & & & \\
\hline \multirow[t]{7}{*}{ Rat } & Salman et al. [31] & GS & 6 weeks & Terencio et al. [44] & $\mathrm{GH}+\mathrm{CS}$ & 12 weeks & & & \\
\hline & Sun et al. [27] & CS & 4 weeks & Panahafir et al. [45] & $\mathrm{GH}$ & 12 weeks & & & \\
\hline & Wang et al. [41] & GS & 4 weeks & Wen et al. [46] & GS & 10 weeks & & & \\
\hline & Ren et al. [42] & CS & 6 weeks & Silva et al. [48] & $\mathrm{GS}+\mathrm{CS}$ & 10 weeks & & & \\
\hline & Sanches et al. [43] & $\mathrm{GH}+\mathrm{CS}$ & 4 weeks & & & & & & \\
\hline & Lee et al. [28] & $\mathrm{GS}+\mathrm{CS}$ & 7 weeks & & & & & & \\
\hline & Naito et al. [47] & GH & 8 weeks & & & & & & \\
\hline Mice & Ivanovska et al. [29] & $\mathrm{GH} / \mathrm{GS}$ & 3 weeks & & & & & & \\
\hline Guinea-Pig & & & & & & & Taniguchi et al. [32] & GH/CS & 18 months \\
\hline
\end{tabular}




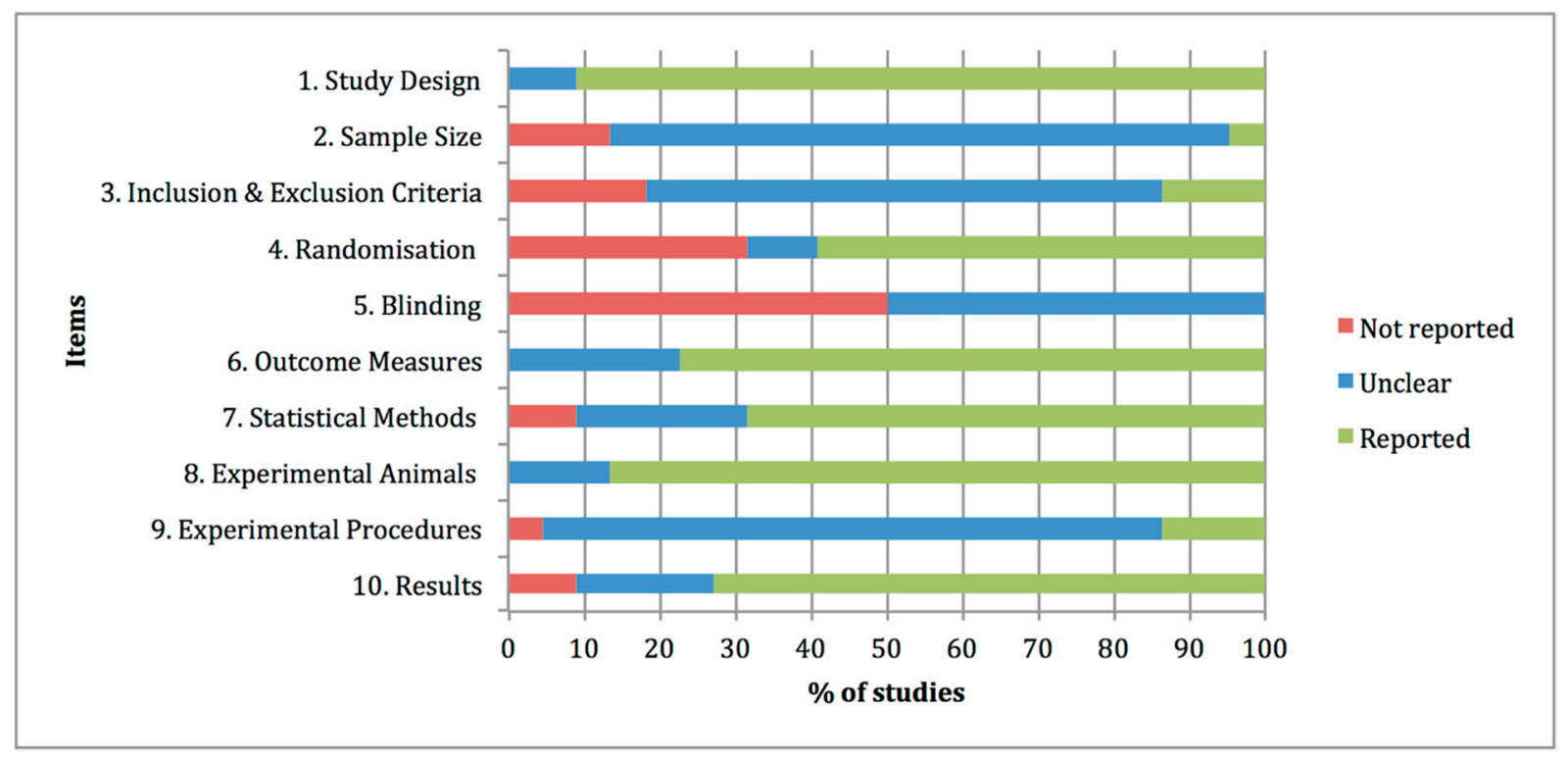

Figure 2. Quality assessments of the 22 preclinical studies included in the systematic review based on the Essential 10 items of the ARRIVE guidelines 2.0. Values are expressed by frequencies (\%).

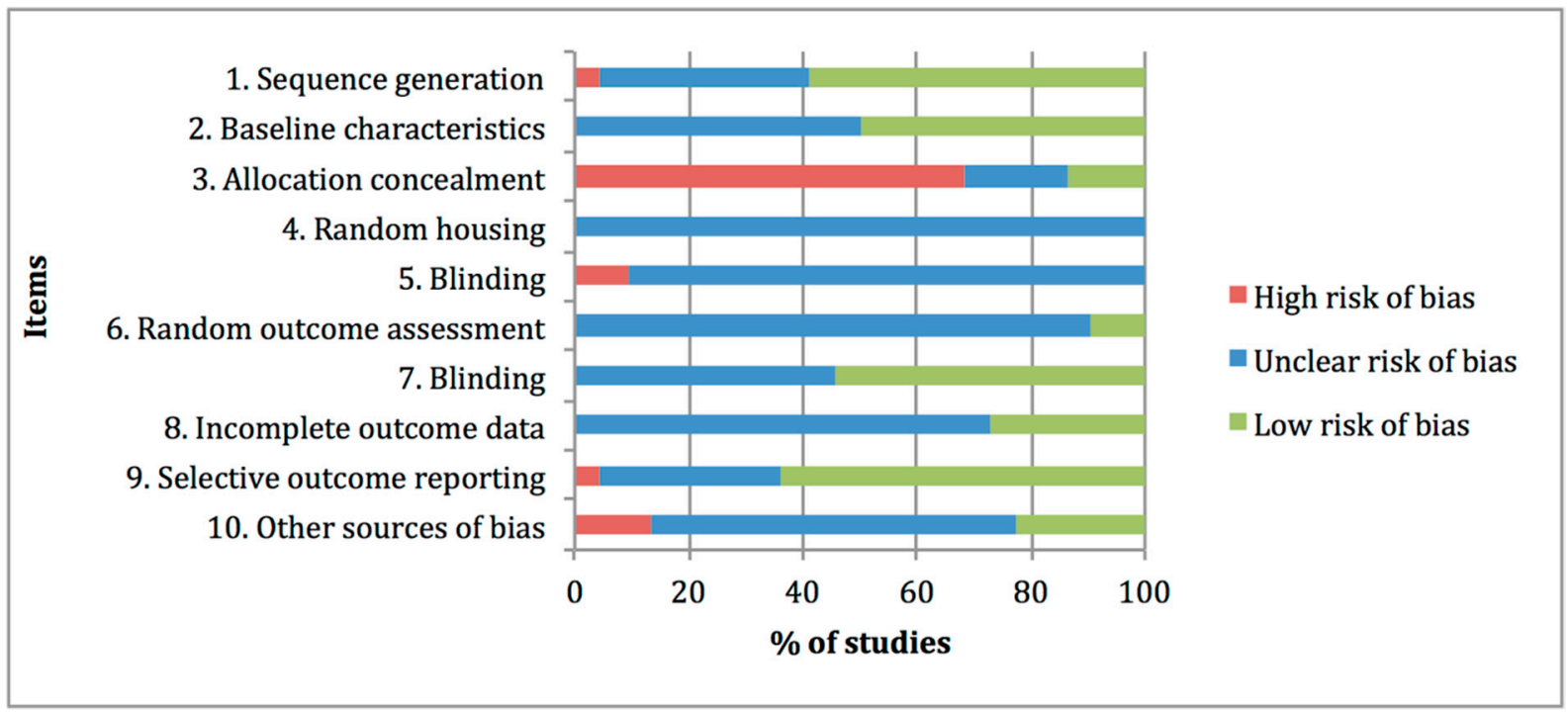

Figure 3. Risk of bias distribution graph of the 22 preclinical studies included in the systematic review according to SYRCLE tool. Values are expressed by frequencies (\%).

\section{Discussion}

The aim of this systematic review was to examine the effect of glucosamine and chondroitin sulfate treatments in the synovial knee joint tissues and specific biomarkers of the osteoarthritic preclinical studies. A total of 22 studies with 3 different types of nutraceuticals: glucosamine sulfate, glucosamine hydrochloride and chondroitin sulfate, administered alone or in different combinations, were meticulously analysed in order to elucidate their direct influence on the main structural and biochemical elements in the OA joints.

Taking into account the experimental animal model, in the studies considered for this review, the most commonly used species were rats and rabbits, whereas only one study employed mouse as animal models [29]. These findings were different from those 
observed by other OA preclinical research, where mouse models constituted the majority of the included studies [49,50]. In regards to OA induction is concerned, in agreement with other publications, surgically induced models were one of the most selected based on the rapid OA induction, repeatability and lower costs [23,51]. Regarding spontaneous models, a slower disease progression was observed and therefore it seems closer to what naturally occurs in primary osteoarthritic disease [52]. However this review included only one study of spontaneous model of OA in guinea pigs [32].

Generally, in this systematic review we found a large inconsistency among the experimental nutraceutical protocols. As an attempt to reduce the variability among studies, we excluded the articles in which animals received intra-articular therapy injections [53-57]. Even though according to the records screened, these local therapies demonstrated a positive chondroprotective effect and anti-inflammatory activity, we decided to evaluate other administrations routes, such as oral and intraperitoneal, in order to analyse the systemic and non-local effects of glucosamine and chondroitin sulfate.

With respect to the therapeutic regimen, there are also notable differences among studies, both in the frequency and in the dosage administered. In this regard, 2 out of 22 articles included in this review examined the efficacy of chondroitin sulfate [27] and glucosamine sulfate [41] at different doses, and both of them concluded that they seemed to reduce the cartilage changes and biomarker alterations in a dose-dependent manner.

Additionally, some articles analysed the potential chondroprotective effect of different nutraceutical combinations. Furthermore, the combination of nutraceutical and other therapies and drugs was also investigated. In this context beneficial effects were observed in the association of glucosamine and risedronate [31,36], glucosamine and fish collagen peptide [37] and glucosamine hydrochloride and chondroitin sulfate plus fursultiamine, where only the combined group with the addition of the vitamin showed a significant chondroprotective effect [39]. Furthermore, in one study using an OA experimental model in rats, enhanced responses were observed with the association of glucosamine sulfate, chondroitin sulfate and photobiomodulation [43].

Regarding the nutraceutical combinations between glucosamine and chondroitin therapies evaluated in this review, Silva et al. [48] observed that the association of glucosamine sulfate and chondroitin sulfate, rather than isolated glucosamine, significantly reduced the joint pain and prevented the cartilage histology alterations. Likewise, Terencio et al. [44] demonstrated the chondroprotective effects of the combination chondroitin sulfate-glucosamine, as well as reduced inflammatory mediator levels. By contrast, RomanBlas et al. [35] did not find any beneficial effects in the combined therapy with chondroitin sulfate plus glucosamine sulfate or hydrochloride. Regarding this point, a study which used a chemically induced murine model, focused on examining the effect of glucosamine on cartilage degradation and bone resorption, comparing two different pharmacological forms, sulfate and hydrochloride; the results showed less histologic effectiveness in the sulfate form when both were administered under the same conditions [29]. It is important to point out that some authors suggested that glucosamine hydrochloride had poorer bioavailability and less beneficial effect in relieving clinical OA symptoms [21]. In our review, slightly higher chondroprotective effects were determined in the glucosamine sulfate studies comparing to the studies that included the glucosamine hydrochloride formulation ( $67 \%$ vs. $50 \%$, respectively). Furthermore, fewer biochemical alterations were found in the glucosamine sulfate administration compared to the hydrochloride ones $(80 \%$ vs. $67 \%$ ). Nevertheless, in a previous review on the use of glucosamine in the management of human OA, the authors determined that, due to the heterogeneous effects observed in the available research studies, concluding which formulation could be more effective continues to be extremely difficult [58].

Another point of interest in the experimental design is the therapy timing initiation in relation to the $\mathrm{OA}$ induction. As previously described, the articles included in this review were grouped into three distinct protocols, pre-emptive, early and delayed administrations. Among these studies, the highest chondroprotective effects were determined in 
the pre-emptive therapies, followed by the delayed ones, which showed slightly positive higher values than the studies with early administrations. These findings were slightly different to those observed in a recently published systematic review about the effect of bisphosphonates therapies in OA preclinical studies [59], where an obvious time-dependent efficacy on cartilage status was determined, showing better chondroprotective effects in pre-emptive and early therapy initiations and greater cartilage damage in the delayed ones. In our opinion, the positive values observed in the delayed administrations could be associated with an inadequate selection of the period of time determined, given that it can be established as early as 14 days after de OA experimental induction and longer periods of time may be required.

In terms of duration, attention is drawn to the lack of evidence in long-term therapy, identifying only 1 out of 22 included publications, in which glucosamine hydrochloride and chondroitin sulfate were evaluated at 8,12 and 18 months, showing reduced cartilage degeneration and biomarker alterations in both treated groups, the animal group treated with glucosamine hydrochloride and the group treated with chondroitin sulfate [32]. In this context, it is important to highlight that the histological and biochemical response after long-term nutraceutical administration is basically unpredictable. The initial positive response identified in some studies may not be sustained for long periods of time. However, the opposite is also possible, and a longer duration of treatment period may be necessary to observe a beneficial effect in the synovial joint. Therefore, additional preclinical studies in OA research evaluating the effect of dietary supplements in the long term are required $[19,59]$.

Overall, in this systematic review, we observed a high variability among the experimental designs. Consequently, making an accurate assessment of how glucosamine and chondroitin sulfate affect the OA progression continues to be a challenge. In general terms, the evaluated nutraceuticals, alone or in combination, did not seem to prevent the subchondral bone changes, the synovial inflammation or the osteophyte formation, showing poor positive responses. Nevertheless, it is true that only a few of the publications included evaluations at those levels. Cartilage continues to be the primary focus in OA research and in this sense, positive chondroprotective effects were identified in approximately half of the publications, the studies of glucosamine sulfate and the combination of chondroitin sulfate plus glucosamine sulfate showing the most promising results. There is also increasing attention on the research of biochemical markers. As it could be observed in this study, they were the second more assessed parameter. In this context, a positive response was identified in more than half of the evaluations included in this review.

Regarding the risk of bias and the quality assessments of the articles included in this review, they were similar to the previous studies $[49,59]$. There are essential details about the experimental design which continue to be poorly reported in the studies, such as the sample size calculation, which was only reported in one of the manuscripts [33]. Inclusion and exclusion criteria were also badly or incompletely reported in most of the studies as well as blinding the experimental details. More specifically, half of the studies did not report the information and the other half only specified it in the outcome assessment stage, but not in the experimental and treatment administration stages. In addition, the information concerning the acclimatisation period of the animals, the housing and husbandry was also insufficient. In this sense, a previous research evaluated the adherence to the ARRIVE checklist in 236 papers between 2009 and 2015, and unexpectedly none of the evaluated manuscripts fully reported $100 \%$ of the items [60]. Consequently, the improvement of the research report in animal experimentation continues to be an essential task at present [25].

To conclude, OA management in companion animals continues to be a challenge in veterinary medicine. As we exposed in this review, glucosamine and chondroitin sulfate seems to provide chondroprotective effects and less inflammatory biochemical response in approximately half of the evaluations. However, these effects are inconsistent between the clinical and the preclinical studies. One explanation may be related to the great variety of histological scoring evaluations, the potential assessor's subjectivity and 
the possibility of intra- and inter-observer variations [61]. Moreover, as these therapies have a slow onset of action, long-term administrations should be required to clarify their effectiveness. Additionally, a possible caregiver placebo effect may explain some of the beneficial responses observed in clinical trials with dogs $[17,62]$. For all these reasons, the use of glucosamine and chondroitin sulfate should be an individual veterinary/owner decision, reached by thoroughly evaluating each particular clinical case and its symptomatic response.

\section{Conclusions}

In this systematic review we found a large inconsistency among the experimental nutraceutical protocols and the outcomes of the studies. Consequently, the comparison among publications evaluating the real effect of glucosamine and chondroitin sulfate on synovial joint tissues and biochemical markers is challenging. The results of this study showed a positive cartilage response and biochemical modulation in approximately half of the articles evaluated. As for the rest of the parameters, these dietary supplements did not appear to adequately supress the subchondral bone changes, the synovial inflammation or the osteophyte formation. However, further experimental studies may be needed to evaluate the nutraceutical effect at those levels. Generally, beneficial effects were associated with a pre-emptive treatment administration, higher doses and multimodality approaches with some combined therapies. Even though some results were promising and encouraging, most of them continue to show a great heterogeneity and at present, there is a need to design high-quality systematic experimental studies. Additional studies focused on long-term treatments, as well as evaluating their potentially disease-modifying effects are required.

Author Contributions: S.F.-M., A.G.-C., F.M., M.G.-G., M.P. and M.L.-P. contributed to the review of the literature and analysis the manuscript. All authors read and critically revised the manuscript. S.F.-M. conceived the idea and drafted the manuscript. All authors have read and agreed to the published version of the manuscript.

Funding: This research received no external funding.

Institutional Review Board Statement: Not applicable.

Data Availability Statement: Not applicable.

Acknowledgments: S.F.-M. acknowledges the FPU programme for her pre-doctoral contract (Ref. FPU15/05325) from the Spanish Government's Ministerio de Ciencia, Innovación y Universidades.

Conflicts of Interest: The authors declare no conflict of interest.

\section{References}

1. Felson, D.T.; Neogi, T. Osteoarthritis: Is It a Disease of Cartilage or of Bone? Arthritis Rheum. 2004, 50, 341-344. [CrossRef]

2. Karsdal, M.A.; Leeming, D.J.; Dam, E.B.; Henriksen, K.; Alexandersen, P.; Pastoureau, P.; Altman, R.D.; Christiansen, C. Should subchondral bone turnover be targeted when treating osteoarthritis? Osteoarthr. Cartil. 2008, 16, 638-646. [CrossRef]

3. Anderson, K.L.; O’Neill, D.G.; Brodbelt, D.C.; Church, D.B.; Meeson, R.L.; Sargan, D.; Summers, J.F.; Zulch, H.; Collins, L.M. Prevalence, duration and risk factors for appendicular osteoarthritis in a UK dog population under primary veterinary care. Sci. Rep. 2018, 8, 1-12. [CrossRef]

4. Rychel, J.K. Diagnosis and Treatment of Osteoarthritis. Top. Companion Anim. Med. 2010, 25, 20-25. [CrossRef]

5. Epstein, M.; Rodan, I.; Griffenhagen, G.; Kadrlik, J.; Petty, M.; Robertson, S.; Simpson, W. 2015 AAHA/AAFP pain management guidelines for dogs and cats. J. Am. Anim. Hosp. Assoc. 2015, 51, 67-84. [CrossRef]

6. Henrotin, Y.; Sanchez, C.; Balligand, M. Pharmaceutical and nutraceutical management of canine osteoarthritis: Present and future perspectives. Vet. J. 2005, 170, 113-123. [CrossRef]

7. Neundorf, R.H.; Lowerison, M.B.; Cruz, A.M.; Thomason, J.J.; McEwen, B.J.; Hurtig, M.B. Determination of the prevalence and severity of metacarpophalangeal joint osteoarthritis in Thoroughbred racehorses via quantitative macroscopic evaluation. Am. J. Vet. Res. 2010, 71, 1284-1293. [CrossRef]

8. Espinosa-Mur, P.; Phillips, K.L.; Galuppo, L.D.; DeRouen, A.; Benoit, P.; Anderson, E.; Shaw, K.; Puchalski, S.; Peters, D.; Kass, P.H.; et al. Radiological prevalence of osteoarthritis of the cervical region in 104 performing Warmblood jumpers. Equine Vet. J. 2020, 1-7. [CrossRef] [PubMed] 
9. Johnston, S.A. Osteoarthritis. Joint anatomy, physiology, and pathobiology. Vet. Clin. N. Am. Small Anim. Pract. 1997, 27, 699-723. [CrossRef]

10. O'Neill, D.G.; Church, D.B.; McGreevy, P.D.; Thomson, P.C.; Brodbelt, D.C. Prevalence of Disorders Recorded in Dogs Attending Primary-Care Veterinary Practices in England. PLoS ONE 2014, 9, e90501. [CrossRef] [PubMed]

11. Slingerland, L.I.; Hazewinkel, H.A.W.; Meij, B.P.; Picavet, P.; Voorhout, G. Cross-sectional study of the prevalence and clinical features of osteoarthritis in 100 cats. Vet. J. 2011, 187, 304-309. [CrossRef]

12. Bennett, D.; bt Zainal Ariffin, S.M.; Johnston, P. Osteoarthritis in the cat: 1 . how common is it and how easy to recognise? J. Feline Med. Surg. 2012, 14, 65-75. [CrossRef] [PubMed]

13. Bennett, D.; bt Zainal Ariffin, S.M.; Johnston, P. Osteoarthritis in the cat: 2. How should it be managed and treated? J. Feline Med. Surg. 2012, 14, 76-84. [CrossRef] [PubMed]

14. Apostu, D.; Lucaciu, O.; Mester, A.; Oltean-Dan, D.; Baciut, M.; Baciut, G.; Bran, S.; Onisor, F.; Piciu, A.; Pasca, R.D.; et al. Systemic drugs with impact on osteoarthritis. Drug Metab. Rev. 2019, 51, 498-523. [CrossRef] [PubMed]

15. Henrotin, Y.; Lambert, C.; Couchourel, D.; Ripoll, C.; Chiotelli, E. Nutraceuticals: Do they represent a new era in the management of osteoarthritis? A narrative review from the lessons taken with five products. Osteoarthr. Cartil. 2011, 19, 1-21. [CrossRef]

16. McCarthy, G.; O’Donovan, J.; Jones, B.; McAllister, H.; Seed, M.; Mooney, C. Randomised double-blind, positive-controlled trial to assess the efficacy of glucosamine/chondroitin sulfate for the treatment of dogs with osteoarthritis. Vet. J. 2007, 174, 54-61. [CrossRef]

17. Scott, R.M.; Evans, R.; Conzemius, M.G. Efficacy of an oral nutraceutical for the treatment of canine osteoarthritis: A doubledblind, randomized, placebo-controlled prospective clinical trial. Vet. Comp. Orthop. Traumatol. 2017, 30, 318-323. [CrossRef] [PubMed]

18. Moreau, M.; Dupuis, J.; Bonneau, N.H.; Desnoyers, M. Clinical evaluation of a nutraceutical, carprofen and meloxicam for the treatment of dogs with osteoarthritis. Vet. Rec. 2003, 152, 323-329. [CrossRef]

19. Comblain, F.; Serisier, S.; Barthelemy, N.; Balligand, M.H. Review of dietary supplements for the management of osteoarthritis indogs in studies from 2004 to 2014. J. Vet. Pharmacol. Ther. 2016, 39, 1-15. [CrossRef]

20. Vandeweerd, J.M.; Coisnon, C.; Clegg, P.; Cambier, C.; Pierson, A.; Hontoir, F.; Saegerman, C.; Gustin, P.; Buczinski, S. Systematic Review of Efficacy of Nutraceuticals to Alleviate Clinical Signs of Osteoarthritis. J. Vet. Intern. Med. 2012, 26, 448-456. [CrossRef]

21. Bhathal, A.; Spryszak, M.; Louizos, C.; Frankel, G. Glucosamine and chondroitin use in canines for osteoarthritis: A review. Open Vet. J. 2017, 7, 36-49. [CrossRef]

22. Mével, E.; Monfoulet, L.E.; Merceron, C.; Coxam, V.; Wittrant, Y.; Beck, L.; Guicheux, J. Nutraceuticals in joint health: Animal models as instrumental tools. Drug Discov. Today 2014, 19, 1649-1658. [CrossRef]

23. Kuyinu, E.L.; Narayanan, G.; Nair, L.S.; Laurencin, C.T. Animal models of osteoarthritis: Classification, update, and measurement of outcomes. J. Orthop. Surg. Res. 2016, 11, 1-27. [CrossRef] [PubMed]

24. de Vries, R.B.M.; Hooijmans, C.R.; Langendam, M.W.; van Luijk, J.; Leenaars, M.; Ritskes-Hoitinga, M.; Wever, K.E. A protocol format for the preparation, registration and publication of systematic reviews of animal intervention studies. Evid. Based Preclin. Med. 2015, 2, e00007. [CrossRef]

25. Percie du Sert, N.; Hurst, V.; Ahluwalia, A.; Alam, S.; Avey, M.T.; Baker, M.; Browne, W.J.; Clark, A.; Cuthill, I.C.; Dirnagl, U.; et al. The ARRIVE guidelines 2.0: Updated guidelines for reporting animal research. PLOS Biol. 2020, 18, e3000410. [CrossRef]

26. Hooijmans, C.R.; Rovers, M.M.; De Vries, R.B.M.; Leenaars, M.; Ritskes-Hoitinga, M.; Langendam, M.W. SYRCLE's risk of bias tool for animal studies. BMC Med. Res. Methodol. 2014, 14, 43. [CrossRef]

27. Sun, Y.; Zhang, G.; Liu, Q.; Liu, X.; Wang, L.; Wang, J.; Liang, L. Chondroitin sulfate from sturgeon bone ameliorates pain of osteoarthritis induced by monosodium iodoacetate in rats. Int. J. Biol. Macromol. 2018, 117, 95-101. [CrossRef] [PubMed]

28. Lee, H.; Park, Y.; Ahn, C.W.; Park, S.H.; Jung, E.Y.; Suh, H.J. Deer bone extract suppresses articular cartilage damage induced by monosodium iodoacetate in osteoarthritic rats: An in vivo micro-computed tomography study. J. Med. Food 2014, 17, 701-706. [CrossRef]

29. Ivanovska, N.; Dimitrova, P. Bone resorption and remodeling in murine collagenase-induced osteoarthritis after administration of glucosamine. Arthritis Res. Ther. 2011, 13. [CrossRef] [PubMed]

30. Torelli, S.R.; Rahal, S.C.; Volpi, R.S.; Sequeira, J.L.; Grassioto, I.Q. Histopathological evaluation of treatment with chondroitin sulphate for osteoarthritis induced by continuous immobilization in rabbits. J. Vet. Med. Ser. A Physiol. Pathol. Clin. Med. 2005, 52, 45-51. [CrossRef] [PubMed]

31. Salman, A.; Shabana, A.I.; El-ghazouly, D.E.S.; Maha, E. Protective effect of glucosamine and risedronate (alone or in combination) against osteoarthritic changes in rat experimental model of immobilized knee. Anat. Cell Biol. 2019, 52, 498-510. [CrossRef]

32. Taniguchi, S.; Ryu, J.; Seki, M.; Sumino, T.; Tokuhashi, Y.; Esumi, M. Long-term oral administration of glucosamine or chondroitin sulfate reduces destruction of cartilage and up-regulation of MMP-3 mRNA in a model of spontaneous osteoarthritis in Hartley guinea pigs. J. Orthop. Res. 2012, 30, 673-678. [CrossRef] [PubMed]

33. Abdul Kadir, A.; Abdul Kadir, A.; Abd Hamid, R.; Mat Jais, A.M.; Omar, J.; Sadagatullah, A.N.; Badrin, S.; Win, T.T.; Sirajudeen, K.N.S.; Salleh, A. Evaluation of Chondroprotective Activity of Channa striatus in Rabbit Osteoarthritis Model. Biomed. Res. Int. 2019, 2019. [CrossRef] [PubMed] 
34. Jeong, D.H.; Ullah, H.M.A.; Goo, M.J.; Ghim, S.G.; Hong, I.H.; Kim, A.Y.; Jeon, S.M.; Choi, M.S.; Elfadl, A.K.; Chung, M.J.; et al. Effects of oral glucosamine hydrochloride and mucopolysaccharide protein in a rabbit model of osteoarthritis. Int. J. Rheum. Dis. 2017, 21, 620-628. [CrossRef]

35. Roman-Blas, J.A.; Mediero, A.; Tardío, L.; Portal-Nuñez, S.; Gratal, P.; Herrero-Beaumont, G.; Largo, R. The combined therapy with chondroitin sulfate plus glucosamine sulfate or chondroitin sulfate plus glucosamine hydrochloride does not improve joint damage in an experimental model of knee osteoarthritis in rabbits. Eur. J. Pharmacol. 2017, 794, 8-14. [CrossRef]

36. Permuy, M.; Guede, D.; López-Peña, M.; Muñoz, F.; Caeiro, J.R.; González-Cantalapiedra, A. Comparison of various SYSADOA for the osteoarthritis treatment: An experimental study in rabbits Clinical rheumatology and osteoporosis. BMC Musculoskelet. Disord. 2015, 16, 1-12. [CrossRef]

37. Ohnishi, A.; Osaki, T.; Matahira, Y.; Tsuka, T.; Imagawa, T.; Okamoto, Y.; Minami, S. Evaluation of the chondroprotective effects of glucosamine and fish collagen peptide on a rabbit ACLT model using serum biomarkers. J. Vet. Med. Sci. 2013, 75, 421-429. [CrossRef]

38. Wang, S.X.; Laverty, S.; Dumitriu, M.; Plaas, A.; Grynpas, M.D. The effects of glucosamine hydrochloride on subchondral bone changes in an animal model of osteoarthritis. Arthritis Rheum. 2007, 56, 1537-1548. [CrossRef]

39. Kobayashi, T.; Notoya, K.; Nakamura, A.; Akimoto, K. Fursultiamine, a vitamin B1 derivative, enhances chondroprotective effects of glucosamine hydrochloride and chondroitin sulfate in rabbit experimental osteoarthritis. Inflamm. Res. 2005, 54, $249-255$. [CrossRef] [PubMed]

40. Tiraloche, G.; Girard, C.; Chouinard, L.; Sampalis, J.; Moquin, L.; Ionescu, M.; Reiner, A.; Poole, A.R.; Laverty, S. Effect of oral glucosamine on cartilage degradation in a rabbit model of osteoarthritis. Arthritis Rheum. 2005, 52, 1118-1128. [CrossRef] [PubMed]

41. Wang, X.X.; Cai, L. Expression level of proteoglycan, collagen and type II collagen in osteoarthritis rat model is promoted and degradation of cartilage is prevented by glucosamine methyl ester. Eur. Rev. Med. Pharmacol. Sci. 2018, 22, 3609-3616. [CrossRef] [PubMed]

42. Ren, Z.; Ji, Y.; Wang, Y.; Dong, L. Chondroitin sulfate from Scophthalmus maximus for treating osteoarthritis. Int. J. Biol. Macromol. 2017, 108, 1158-1164. [CrossRef] [PubMed]

43. Sanches, M.; Assis, L.; Criniti, C.; Fernandes, D.; Tim, C.; Renno, A.C.M. Chondroitin sulfate and glucosamine sulfate associated to photobiomodulation prevents degenerative morphological changes in an experimental model of osteoarthritis in rats. Lasers Med. Sci. 2017, 33, 549-557. [CrossRef]

44. Terencio, M.C.; Ferrándiz, M.L.; Carceller, M.C.; Ruhí, R.; Dalmau, P.; Vergés, J.; Montell, E.; Torrent, A.; Alcaraz, M.J. Chondroprotective effects of the combination chondroitin sulfate-glucosamine in a model of osteoarthritis induced by anterior cruciate ligament transection in ovariectomised rats. Biomed. Pharmacother. 2016, 79, 120-128. [CrossRef]

45. Panahifar, A.; Jaremko, J.L.; Tessier, A.G.; Lambert, R.G.; Maksymowych, W.P.; Fallone, B.G.; Doschak, M.R. Development and reliability of a multi-modality scoring system for evaluation of disease progression in pre-clinical models of osteoarthritis: Celecoxib may possess disease-modifying properties. Osteoarthr. Cartil. 2014, 22, 1639-1650. [CrossRef] [PubMed]

46. Wen, Z.H.; Tang, C.C.; Chang, Y.C.; Huang, S.Y.; Hsieh, S.P.; Lee, C.H.; Huang, G.S.; Ng, H.F.; Neoh, C.A.; Hsieh, C.S.; et al. Glucosamine sulfate reduces experimental osteoarthritis and nociception in rats: Association with changes of mitogen-activated protein kinase in chondrocytes. Osteoarthr. Cartil. 2010, 18, 1192-1202. [CrossRef]

47. Naito, K.; Watari, T.; Furuhata, A.; Yomogida, S.; Sakamoto, K.; Kurosawa, H.; Kaneko, K.; Nagaoka, I. Evaluation of the effect of glucosamine on an experimental rat osteoarthritis model. Life Sci. 2010, 86, 538-543. [CrossRef] [PubMed]

48. Silva, F.S.; Yoshinari, N.H.; Castro, R.R.; Girão, V.C.C.; Pompeu, M.M.L.; Andrade Feitosa, J.P.; Rocha, F.A.C. Combined glucosamine and chondroitin sulfate provides functional and structural benefit in the anterior cruciate ligament transection model. Clin. Rheumatol. 2009, 28, 109-117. [CrossRef]

49. Contartese, D.; Tschon, M.; De Mattei, M.; Fini, M. Molecular Sciences Sex Specific Determinants in Osteoarthritis: A Systematic Review of Preclinical Studies. Int. J. Mol. Sci. 2020, 21, 3696. [CrossRef] [PubMed]

50. Shmagel, A.; Demmer, R.; Knights, D.; Butler, M.; Langsetmo, L.; Lane, N.E.; Ensrud, K. The effects of glucosamine and chondroitin sulfate on gut microbial composition: A systematic review of evidence from animal and human studies. Nutrients 2019, 11, 294. [CrossRef]

51. Lampropoulou-Adamidou, K.; Lelovas, P.; Karadimas, E.V.; Liakou, C.; Triantafillopoulos, I.K.; Dontas, I.; Papaioannou, N.A. Useful animal models for the research of osteoarthritis. Eur. J. Orthop. Surg. Traumatol. 2014, 24, 263-271. [CrossRef] [PubMed]

52. McCoy, A.M. Animal Models of Osteoarthritis: Comparisons and Key Considerations. Vet. Pathol. 2015, 52, 803-818. [CrossRef]

53. Fonsi, M.; El Amrani, A.I.; Gervais, F.; Vincent, P. Intra-Articular Hyaluronic Acid and Chondroitin Sulfate: Pharmacokinetic Investigation in Osteoarthritic Rat Models. Curr. Ther. Res. Clin. Exp. 2020, 92. [CrossRef]

54. Frisbie, D.D.; McIlwraith, C.W.; Kawcak, C.E.; Werpy, N.M. Evaluation of intra-articular hyaluronan, sodium chondroitin sulfate and $\mathrm{N}$-acetyl-d-glucosamine combination versus saline $(0.9 \% \mathrm{NaCl})$ for osteoarthritis using an equine model. Vet. J. 2013, 197, 824-829. [CrossRef]

55. Gibson, M.; Li, H.; Coburn, J.; Moroni, L.; Nahas, Z.; Bingham, C.; Yarema, K.; Elisseeff, J. Intra-articular delivery of glucosamine for treatment of experimental osteoarthritis created by a medial meniscectomy in a rat model. J. Orthop. Res. 2014, 32, 302-309. [CrossRef] 
56. Scotto D’Abusco, A.; Corsi, A.; Grillo, M.G.; Cicione, C.; Calamia, V.; Panzini, G.; Sansone, A.; Giordano, C.; Politi, L.; Scandurra, R. Effects of intra-articular administration of glucosamine and a peptidyl-glucosamine derivative in a rabbit model of experimental osteoarthritis: A pilot study. Rheumatol. Int. 2008, 28, 437-443. [CrossRef] [PubMed]

57. Shikhman, A.R.; Amiel, D.; D'Lima, D.; Hwang, S.B.; Hu, C.; Xu, A.; Hashimoto, S.; Kobayashi, K.; Sasho, T.; Lotz, M.K Chondroprotective activity of $\mathrm{N}$-acetylglucosamine in rabbits with experimental osteoarthritis. Ann. Rheum. Dis. 2005, 64, 89-94. [CrossRef]

58. Henrotin, Y.; Mobasheri, A.; Marty, M. Is there any scientific evidence for the use of glucosamine in the management of human osteoarthritis? Arthritis Res. Ther. 2012. [CrossRef] [PubMed]

59. Fernández-Martín, S.; López-Peña, M.; Muñoz, F.; Permuy, M.; González-Cantalapiedra, A. Bisphosphonates as disease-modifying drugs in osteoarthritis preclinical studies: A systematic review from 2000 to 2020. Arthritis Res. Ther. 2021, 23, 60. [CrossRef]

60. Leung, V.; Rousseau-Blass, F.; Beauchamp, G.; Pang, D.S.J. ARRIVE has not ARRIVEd: Support for the ARRIVE (Animal Research: Reporting of in vivo Experiments) guidelines does not improve the reporting quality of papers in animal welfare, analgesia or anesthesia. PLoS ONE 2018, 13, e0197882. [CrossRef]

61. Pastoureau, P.C.; Hunziker, E.B.; Pelletier, J.P. Cartilage, bone and synovial histomorphometry in animal models of osteoarthritis. Osteoarthr. Cartil. 2010, 18, S106-S112. [CrossRef] [PubMed]

62. Malek, S.; Sample, S.J.; Schwartz, Z.; Nemke, B.; Jacobson, P.B.; Cozzi, E.M.; Schaefer, S.L.; Bleedorn, J.A.; Holzman, G.; Muir, P. Effect of analgesic therapy on clinical outcome measures in a randomized controlled trial using client-owned dogs with hip osteoarthritis. BMC Vet. Res. 2012, 8, 185. [CrossRef] [PubMed] 